\title{
Management of renal Stones for the non-specialist
}

\begin{abstract}
Abbreviations: PUJ, pelvico-ureteric junction; AAA, abdominal aortic aneurysm; NSAIDS, non-steroidal antiinflammatory; PCNL, percutaneous nephrolithotomy; SWL, shockwave lithotripsy; URS, ureterorenoscopy
\end{abstract}

\section{Introduction}

The incidence and prevalence of renal stones has increased significantly over the past decade, carrying an individual lifetime risk of $8-10 \%{ }^{1}$ and 83000 UK hospital attendances annually. ${ }^{2}$ With this trend likely to continue initial diagnosis, investigations and management of simple renal stones are increasingly performed by the non-specialist and so it is important for them to be aware of the latest evidence. We review the latest evidence for management below.

\section{Risk factors}

Risk factors for stone formation are categorized into intrinsic and extrinsic causes. Intrinsic causes include age, family history and sex. The peak age of presentation is 40-60 years old. Patients with a first degree relative with renal stones have an increased risk (RR 2.5). ${ }^{3}$ The relative risk between men and women has narrowed over the past decade from 3:1 to 1.3:1 (ibid). Obesity, fluid intake and diet are the most important extrinsic risk factors. There is a strong correlation between BMI and stone formation. A BMI of $>27.5$ doubles the relative risk compared with a BMI of $20-22.5 .{ }^{4}$ Fluid intake is inversely correlated with stone formation. Consuming $>2500 \mathrm{mls} /$ day halves the relative risk compared with $<1250 \mathrm{mls} /$ day. ${ }^{5}$ A low meat intake $(<50 \mathrm{~g} /$ day $)$ halves the risk of stone formation compared with a high meat intake $(>100 \mathrm{~g} /$ day $) .{ }^{4}$ Perversely a low, rather than high, calcium diet predisposes to calcium stone formation. ${ }^{5}$

\section{Presentation}

The majority of renal stones remain asymptomatic. Up to $5 \%$ of abdominal ultrasounds and CTs find incidental renal stones. ${ }^{6}$ Small non-obstructing stones within the kidney are typically asymptomatic or have mild symptoms. The most common presentation of a renal stone is loin pain caused by ureteric obstruction. The classical presentation is severe colicky pain which reaches its peak within 1-2 hours and then becomes constant. Hospital presentation within 12 hours of onset of the pain is a significant predictor of renal colic. ${ }^{7}$ The location of the stone within the ureter determines the nature of the pain. The most common sites are the pelvico-ureteric junction (PUJ), the pelvic brim and vesico-ureteric junction. PUJ stones typically present with a deep loin pain without radiation, pelvic brim stones with flank or lower quadrant pain radiating into the groin and vesico-ureteric stones with irritative voiding symptoms. Flank pain is not specific to renal stones with stones being demonstrated on CT in $34-73 \%$ of these patient. ${ }^{8}$ Nausea, vomiting and a change in appetite are present in approximately $50 \%$ of cases. ${ }^{7}$ Other less common symptoms of renal stones include visible haematuria, recurrent urinary tract infections and the painless passage of grit. On examination, loin tenderness may be present in up to $86 \%$ of patients. ${ }^{7}$ Flank and iliac fossa tenderness may be palpated, but there should be no signs of peritonitis. A thorough examination

\author{
Volume 2 Issue 2 - 2018
}

\author{
R Menzies-Wilson, S Folkard and B Somani \\ Epsom and St Helier University Hospital NHS Trust, UK
}

Correspondence: Richard Menzies-Wilson, Epsom and St Helier University Hospital NHS Trust, UK,

Email rmenzieswilson@gmail.com

Received: February 23, 2018 | Published: March 06, 2018

of patient should be performed to rule out the red flags and other differentials mentioned below.

\section{Investigations}

Detection of haematuria on urine dipstick is the most discriminating bedside investigation. It has a sensitivity for renal stones of $84 \%$, specificity of $48 \%$, PPV of $72 \%$ and NPV of $65 \%{ }^{8}$ It should be noted that the sensitivity is $95 \%$ on day 0 from the onset of pain but decreases to $65 \%$ by day $3 .{ }^{9}$ Low dose non-contrast-enhanced CT (CT KUB) is the gold-standard investigation for most adults, with a sensitivity and specificity of $95 \%$ and $98 \%{ }^{9,10}$ All renal stone types are detectable by CT KUB except for Indinavir stones. These rare stones form as a consequence of the medication Indinavir Phosphate, used in HIV management. ${ }^{11}$ Non-contrast-enhanced computed tomography can determine stone density, inner structure of the stone and the surrounding anatomy. ${ }^{8-11}$ These parameters can affect the selection of treatment modality as we discuss later. Patients with a BMI $>30$ should have a standard-dose non-contrast-enhanced CT to maintain sensitivity and specificity at $95 \%$ and $98 \% .{ }^{10}$ Ultrasonography is the first line investigation for pregnant patients. ${ }^{8}$ This has a sensitivity and specificity of $84 \%$ and $53 \%$. It is important to be aware that normal pregnancy changes can mimic ureteric obstruction (ibid). Magnetic Resonance Imaging can be used as a second line investigation to visualise the level of obstruction and anatomical characteristics. ${ }^{8}$ Ultrasonography should also be performed first line for patients under 16 years old. ${ }^{8}$ This can be performed in conjunction with plain film KUB to increase the sensitivity and specificity. Cystine and Struvite stones, which together make up $10-16 \%$ of stones, are poorly visualised on a plain film and small stones are often missed. ${ }^{8}$ Consequently, the sensitivity and specificity of plain KUB film is $57 \%$ and $76 \%$ (ibid). If ultrasonography and plain-film are inconclusive then low-dose noncontrast CT can be considered (ibid). Blood tests are useful to both rule out other differential diagnoses and complications of renal stones (highlighted below).

\section{Differential diagnosis}

Other renal causes of acute flank pain include: pyelonephritis (typically fevers, rigors and vomiting with infection evident on urine dipstick); blood clot ureteric obstruction (secondary to frank 
haematuria); pelvico-ureteric junction obstruction (classically after consuming large fluid volumes causing dilation of the renal pelvis); urological tumor (which more usually a chronic pain) and renal infarct (classically with fevers, vomiting and a history of atrial fibrillation). Non-renal pathologies attribute for approximately $10 \%$ of acute loin pain of these $37 \%$ are gynecological (with ovarian cysts being the most common), $24 \%$ diverticulitis and $15 \%$ appendicitis ${ }^{12}$ which can also present with microscopic haematuria. Other pathologies include pancreatitis, biliary colic and cholecystitis. Symptomatic Abdominal Aortic Aneurysm (AAA) is a less common but a serious differential diagnosis. One case series reported $18 \%$ of symptomatic leaking AAA's being misdiagnosed as renal colic. ${ }^{13}$ Clinically $80 \%$ of these cases had a palpable and expansile abdominal mass and all patients were $>60$ years old (ibid). Aortic dissection or rupture is poorly demonstrated by non-contrast $\mathrm{CT}$ and so early contrast CT or ultrasound is indicated. Musculoskeletal pain is commonly misdiagnosed as renal colic. This is most commonly mechanical backpain but less-common pathologies include: thoracolumbar vertebral fractures, metastases, and multiple myeloma and psoas haematomas/ collections.

\section{Red flags - Urgent Urology Referral}

The following are indications for an urgent admission:

\section{Obstructed, infected kidney:}

1. Sepsis must be ruled out both clinically and through investigations. Fever, rigors, deranged observations, a White Cell Count of $>15,000 / \mu \mathrm{L}$ or leukocytes/nitrites on urine dipstick are suggestive.

\section{Deranged Renal Function:}

2. If the renal function is acutely impaired, it suggests renal obstruction with abnormal function of contralateral kidney.

\section{Single functioning kidney:}

3. Urgent decompression is required of an obstructed solitary normal functioning kidney.

\section{Uncontrolled pain:}

4. Pain non controlled with oral/per rectal analgesics require admission for intravenous analgesia.

\section{Immediate (non-specialist) management}

Analgesia and antiemetics are the first step of management for patients. Per-rectal non-steroidal anti-inflammatory (NSAIDS) medications (such as diclofenac $150 \mathrm{mg}$ /day 3-10 days) have the best efficacy and should be used as first line European Association of Urology, 2015). Contraindications for diclofenac include congestive heart failure, ischaemic heart disease and peripheral arterial- and cerebrovascular disease and poor renal function. Mild opioids should be used as second line (ibid). Parenteral analgesia and antiemetics should be used for rapid control of severe pain or nausea. Evidence of the use of alpha-blockers (such as tamsulosin) is controversial but European guidelines suggest using them to improve pain and ease passage (ibid). There is no clear evidence to support the use of antispasmodics for pain control (ibid).

\section{Emergency management of ureteric stones}

The emergency decompression of the renal system for the indications above can be achieved by ureteric stenting or nephrostomy. A double-J ureteric stent is passed retrogradely under general anaesthetic and fluoroscopy to relieve the obstruction. General anaesthetic can be difficult if the patient is very unwell or has unfavourable co-morbidities. There is also a failure rate associated with an impacted ureteric stone. ${ }^{14}$ Nephrostomy involves passing a drain through the skin under radiology guidance into the renal pelvis. A stent can then be introduced anterogradely if required. This requires a skilled interventional radiologist, but can be done under local anaesthetic. There is a risk of injury to adjacent organs and the requirement for a nephrostomy bag, ${ }^{15}$ which can be difficult to manage in confused or uncooperative patients. These are temporizing measures, and the stone must then be dealt with definitely which often is attempted at a few weeks post acute admission.

\section{Patient advice with renal stones who do not need admission}

a. Seek medical advice urgently if your pain is not under control or you have a fever.

b. Measures to reduce stone formation ${ }^{16-18}$

c. Drink enough to produce a urine output of 2 to $2 \frac{1}{2}$ litres (4 to 5 pints) each day. It is best to drink continuously throughout the day, rather than going from periods of dehydration to drinking lots in one go.

d. Do not restrict your calcium intake, as a low calcium diet actually increases your risk of stone formation.

e. Reduce intake of animal protein (especially meat), refined sugar and salt.

\section{Non-emergency further management}

The options for further management depend on the size, site, and composition of the stone. Options include conservative management, shock-wave lithotripsy (SWL), ureterorenoscopy (URS), or percutaneous nephrolithotomy (PCNL). SWL is a day-case procedure which focuses ultrasound waves from outside the body onto a stone to fragment the stone. It may be uncomfortable and analgesia is usually given beforehand. Common complications include pain, need for further therapy (which may be operative), failure, infection; bruising or blistering of the skin and precipitation of ureteric colic from stone fragments. ${ }^{17}$ URS involves the insertion of ureteroscope under a general anaesthetic. The stone may then be retrieved using a variety of devices, or laser fragmented to smaller fragments or dust. A ureteric stent can also be inserted at the same time if required. Complications include: mucosal injury, false passage, ureteric perforation, bleeding, extra-ureteric stone migration, fever or sepsis, colic from haematuria and rarely ureteric avulsion. ${ }^{15}$ PCNL uses a nephroscope under general anaesthetic to access the kidney through a loin incision. The stone is broken up with laser and pneumatic energy and usually removed through the nephroscopy tract. This usually necessitates a 2-5 day stay in hospital, a nephrostomy bag and a urethral catheter. These can be removed in the absence of complications when post-operative imaging shows adequate stone removal. ${ }^{18}$ All patients can be expected to experience some haematuria with a risk of blood transfusion in up to $5 \%$ of cases. Other complications include UTI and urosepsis and 
further auxiliary procedures if the stone clearance is inadequate. Open surgery is very rarely used with the advent of the minimally-invasive techniques described above, and is usually reserved for very large stones or abnormal renal anatomy. ${ }^{19}$

\section{Decision-making for surgical options}

Stones less than $4 \mathrm{~mm}$ have an $80 \%$ chance of passage..$^{20}$ Two thirds of stones less than $7 \mathrm{~mm}$ can be expected to pass within four weeks. ${ }^{21}$ A 2014 Cochrane review examined the efficacy of SWL against PCNL and URS for urinary tract stones in general requiring further management. It concluded, noting only five randomised controlled trials of low methodological quality, that SWL is less effective for renal stones than PCNL. This was based on success of treatment at three months (RR $0.46,95 \%$ CI 0.35 to 0.62 ). One RCT found no significant difference between SWL and URS using the same outcome measure (RR $0.91,95 \%$ CI 0.64 to 1.30 ). ${ }^{22}$

\section{Stones in the kidney}

Factors affecting SWL success include size of stone, composition, anatomy and body habitus. It is contra-indicated in pregnant and anticoagulated patients. In a meta-analysis covering 2927 patents in 13 centres, a $90 \%$ clearance rate was calculated for upper and mid-pole stones, and $59 \%$ clearance for lower pole stones. Of these, stones less than $10 \mathrm{~mm}$ had a $74 \%$ clearance rate; those $11-20 \mathrm{~mm} 56 \%$; and those greater than $20 \mathrm{~mm} 33 \%$. Cystine stones generally do not respond well to lithotripsy. Infundibular length, width and infundibulopelvic angle have been shown to influence SWL success ${ }^{20}$, and obesity limits efficacy. Percussion, diuresis and inversion have been shown to increase its success. ${ }^{23}$ Therefore, upper pole renal stones if less than $20 \mathrm{~mm}$ deserve a trial of SWL as initial management. Stones greater than $20 \mathrm{~mm}$ will usually require PCNL. Given the limitations of SWL with lower pole renal stones, it can be recommended as initial treatment in stones less than $10 \mathrm{~mm}$. It is unclear whether URS, SWL or PCNL is best for stones sized $10 \mathrm{~mm}-20 \mathrm{~mm}$, but for lower pole stones $20 \mathrm{~mm}$ and above, PCNL can be recommended (Raman JD, 2008). Staghorn calculi, which involve the renal pelvis and several or all of the calyces are best dealt with by PCNL. ${ }^{24}$

\section{Stones in the ureter}

Treatment options are SWL or URS. European and American studies have agreed on success rates for SWL for ureteric stones, with a stone-free rate of $83.5 \%$, and a retreatment rate of $10.7 \% .^{25}$ Current guidance suggests that for proximal stones, SWL is slightly superior to URS for stones $<10 \mathrm{~mm}$ diameter. For mid-ureteric stones there is clinical equipoise. For distal stones, URS is favored. ${ }^{26}$ These recommendations were based on an index patient: non-pregnant, with a unilateral non-cystine/non-uric acid radio-opaque stone in absence of renal stones needing treatment, with normal contralateral kidney and medical history, normal body habitus and favourable anatomy. ${ }^{27-30}$

\section{Conclusion and key points}

Renal stones are common and an understanding of the basics of presentation, investigations and management is essential for the generalist. Whilst initial presentation is most commonly loin or flank pain with non-visible haematuria, alternate presentations are not uncommon and urine dipstick has its limitations. Low-dose non-contrast $\mathrm{CT}$ is the gold standard investigation for suspected renal stones in an adult with a $\mathrm{BMI}<30$. An obstructed kidney with deranged function or infection is an emergency requiring urgent decompression. Non-emergency management of renal stones includes conservative management, extra-corporeal shock-wave lithotripsy (SWL), ureterorenoscopy (URS), or percutaneous nephrolithotomy (PCNL). This choice depends on patient and stone factors with joint decision making after patient counseling.

\section{Acknowledgements}

None.

\section{Conflict of interest}

The author declares there is no conflict of interest.

\section{References}

1. Romero V, Akpinar H, Assimos D. Kidney stones: a global picture of prevalence, incidence, and associated risk factors. Reviews in urology. 2010;12(2-3):86-96.

2. Turney B, Reynard J, Noble J, et al. Trends in urological stone disease. BJU international. 2012;109(7):1082-1087.

3. Scales C, Curtis L, Norris R, et al. Changing gender prevalence of stone disease. The Journal of urology. 2007;177(3):979-982.

4. Oxford Stone Group. Epidemiology; 2017.

5. Curhan G, Willett W, Rimm E, et al. A prospective study of dietary calcium and other nutrients and the risk of symptomatic kidney stones. The New England journal of medicine. 1993;328(12):833838 .

6. Varrier M, Willis S, Ostermann M. Renal colic-Clinical Review. 2013.

7. Eskelinen M, Ikonen J, Lipponen P. Usefulness of history-taking, physical examination and diagnostic scoring in acute renal colic. European urology. 1998;34(6):467-473.

8. Bultitude M, Smith D, Thomas K. Contemporary Management of Stone Disease: The New EAU Urolithiasis Guidelines for 2015. European urology. 2016;69(3):483-484.

9. Kobayashi T, Nishizawa K, Mitsumori K, et al. Impact of date of onset on the absence of hematuria in patients with acute renal colic. The Journal of urology. 2003;170(4):1093-1096.

10. Brisbane W, Bailey M, Sorensen M. An overview of kidney stone imaging techniques. Nature reviews Urology. 2016;13(11):654-662.

11. Wu D, Stoller M. Indinavir urolithiasis. Current opinion in urology. 2000;10(6):557-561.

12. Abedin A. Managing acute renal colic in primary care. Stourbridge. 2017:1-27.

13. Borrero E, Queral L. Symptomatic abdominal aortic aneurysm misdiagnosed as nephroureterolithiasis. Annals of vascular surgery. 1998;2(2):145-149.

14. Pearle M, Pierce H, Miller G, et al. Optimal method of urgent decompression of the collecting system for obstruction and infection due to ureteric calculi. J Urol. 1998;160:1260-1264.

15. Arya M, Shergill I, Muneer A. et al. Viva Practice for the FRCS (Urol) Examination. Abingdon: Radcliffe Publishing Ltd. 2010.

16. BAUS. BAUS ESWL patient guidance; 2017. 
17. BAUS. BAUS Patient Guidance; 2017.

18. BAUS. Patient Leaflet PCNL; 2017.

19. NHS. Kidney Stones- Treatment; 2016.

20. Walton T. Management of renal and ureteric stones (BAUS Open Resource). 2011

21. Hubner. Natural history and current concepts for the treatment of small ureteral calculi. Eur Urol. 1993;24(2):172-176.

22. Cochrane. Extracorporeal shock wave lithotripsy (ESWL) versus percutaneous nephrolithotomy (PCNL) or retrograde intrarenal surgery (RIRS) for kidney stones. Cochrane library. 204.

23. Pace K, Tariq N, Dyer S, et al. Mechanical percussion, inversion and diuresis for residual lower pole fragments after shock-wave lithotripsy: a prospective, single-blind, randomised controlled trial. J Urol. 2001;166:2072-2080.

24. Preminger G. Diagnosis and acute management of suspected nephrolithiasis in adults. 2016
25. Mobley TB, Myers DA, Grine WB, et al. Low energy lithotripsy with the Lithostar: treatment results with 19,962 renal and ureteral calculi. J Urol. 1994;149(6):1419-1424.

26. Preminger GM, Tiselius HG, Assimos DG, et al. 2007 Guideline for the management of ureteric calculi. Eur Urol. 2007;52(6):1610 1631 .

27. Lingeman JE, Siegel YI, Steele B, et al. Management of lower pole nephrolithiasis: a critical analysis. J Urol. 1994;151(3):663-766.

28. European Association of Urology. Urolithiasis. 2015.

29. Raman JD. Management options for lower pole renal calculi. Curr Opin Urol. 2008;18(2):214-219.

30. Thomas M. Caring for Australians with Renal Impairment (CARI). The CARI guidelines: Clinical diagnosis of kidney stones. Nephrology. 2007;12(1):1-3. 